\title{
Study on the hydrodynamic characters of high-speed water-entry projectiles with different shapes
}

\author{
Wen-qing SHI ${ }^{1, \text { a }}$, Shun-ping LI ${ }^{1, \mathrm{~b}}$, Kai SUN ${ }^{1, \mathrm{c}}$, Guo-jie $\mathrm{LI}^{1, \mathrm{~d}}$, Liang QIAO ${ }^{1, \mathrm{e}}$ and \\ Pu-guang LUO ${ }^{1, f}$
}

1Beijing Institute of space long march vehicle, Beijing 100076 , China

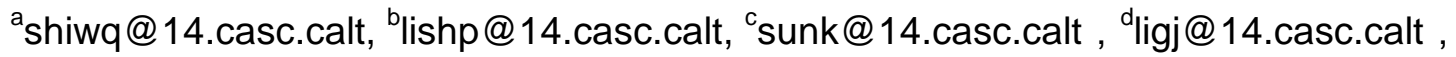
eqiaol@14.casc.calt , 'luopg@14.casc.calt

Keywords: numerical simulation, hydrodynamic characters, penetrate water with high-speed Abstract. The hydrodynamic characteristics, such as the displacement, velocity, acceleration, resistance coefficient and attack angle of three kinds of projectiles are analyzed by numerical simulation. The small taper angle projectile has the smallest resistance coefficient and attack angle. The results of this study can provide some reference for the hydrodynamic shape design of the projectile penetrating water with high-speed.

\section{Introduction}

Hydrodynamic shape has siganature influence on the water-entry trajectory stability and velocity attenuation[1]-[4], which is related to whether the projectile can move to the intended position to play the damage effect. When the projectile penetrates the water free surface at a certain velocity, a series of complex flow phenomenon will form at the projectile nose and its surrounding water. One of the most important is cavity forming around the projectile below the water surface. With having important influence on the water-entry trajectory stability and velocity, water-entry cavity is an important factor that must be taken into account in the hydrodynamic shape design of the projectile.

In this paper, the ball-cone-column projectile, the small taper angle projectile and the column projectile penetrating the water with high-speed are numerically simulated and compared.

\section{The hydrodynamic shape of the projectiles}

The shape of the projectile, especially the shape of the nose, directly affects the characteristics of the the projectile entering the water with high-speed. The structure dimensions of the three kinds of projectiles are shown in Fig. 1, Fig. 2 and Fig.3.

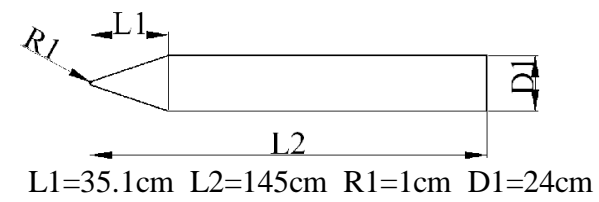

Figure. 1 The structure diagram of the ball-cone-column projectile

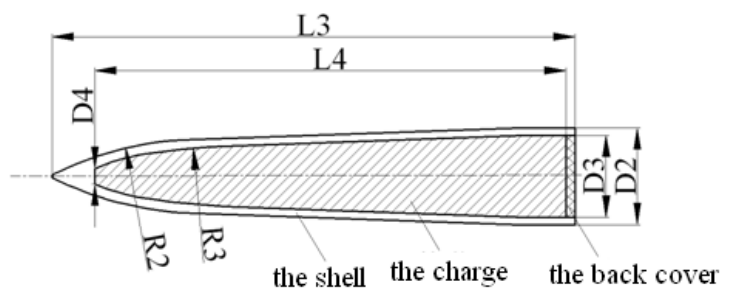

$\mathrm{L} 3=220 \mathrm{~cm} \quad \mathrm{~L} 4=198.2 \mathrm{~cm} \mathrm{R} 2=143.7 \mathrm{~cm} \mathrm{R} 3=140.7 \mathrm{~cm} \mathrm{D} 2=43.2 \mathrm{~cm} \mathrm{D} 3=36 \mathrm{~cm}$ D $4=7.2 \mathrm{~cm}$

Figure. 2 The structure diagram of the small taper angle projectile 


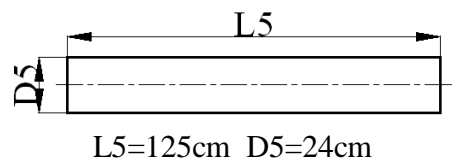

Figure. 3 The structure diagram of the column projectile

\section{Contrastive analysis of dynamic characteristics of the projectiles penetrating water with high-speed}

The processes of the projectiles penetrating into the water at a velocity of $700 \mathrm{~m} / \mathrm{s}$ and an angle of 20 are simulated.

The curves of the velocity with time of the three kinds of projectiles are shown in Fig. 4. During the $50 \mathrm{~ms}$ simulation time, the velocity of the projectile decreases, and the curve exhibits a certain fluctuation, which is the result of the interaction between the shell and the water.

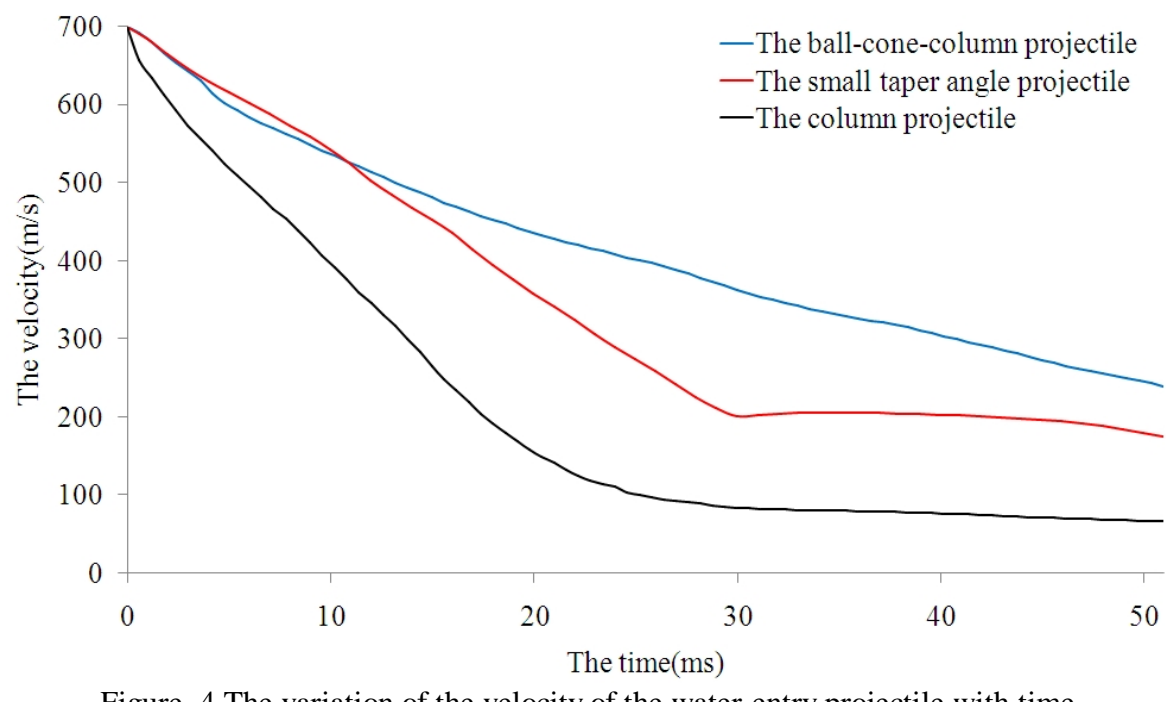

Figure. 4 The variation of the velocity of the water-entry projectile with time

As shown in Fig. 5, in the process of 50ms, the acceleration of the three projectile are very large before $20 \mathrm{~ms}$, then gradually reduce to zero.

The resistance coefficient of the projectile is an important parameter to reflect the motion coefficient. In this paper, the resistance coefficient is calculated by numerical simulation data. The projectile is subjected to gravity and water resistance during penetrating into the water, according to Newton's second law,

$$
m a=m g-C \frac{1}{2} \rho v^{2} S
$$

Where $\mathrm{m}$ is the mass of the projectile, $\mathrm{a}$ is the acceleration of the projectile, $\mathrm{g}$ is the acceleration of gravity, $\rho$ is the density of water, $v$ is the velocity of the projectile, and $S$ is the cross-sectional area of the projectile.

The resistance coefficient is calculated as follows:

$$
C=\frac{-m a+m g}{\frac{1}{2} \rho v^{2} S}
$$




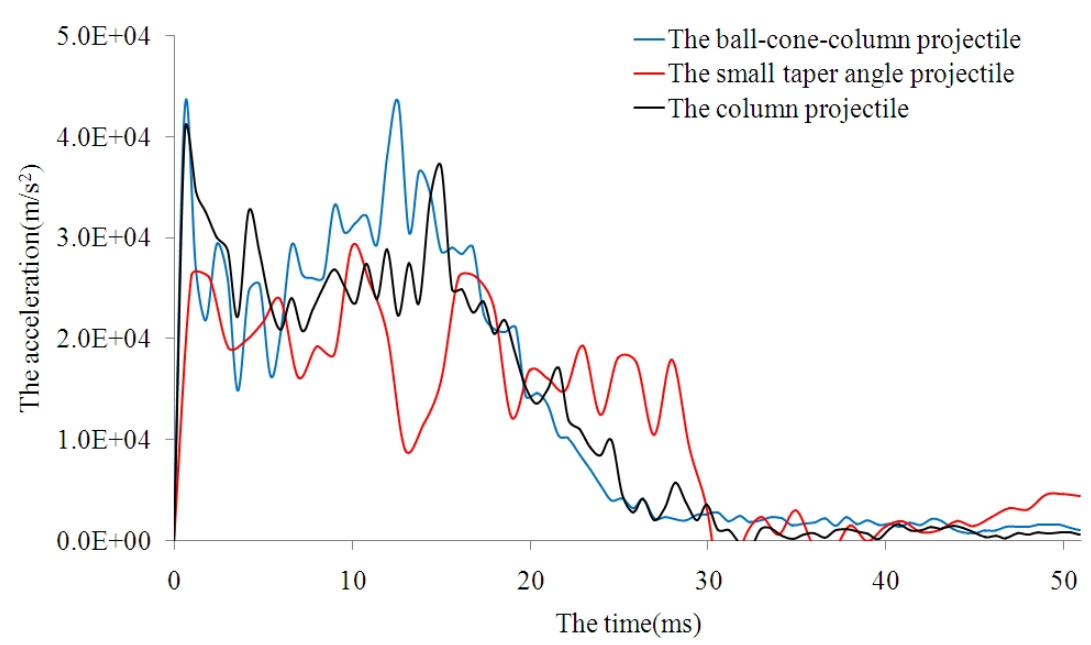

Figure. 5 The variation of the acceleration of the water-entry projectile with time

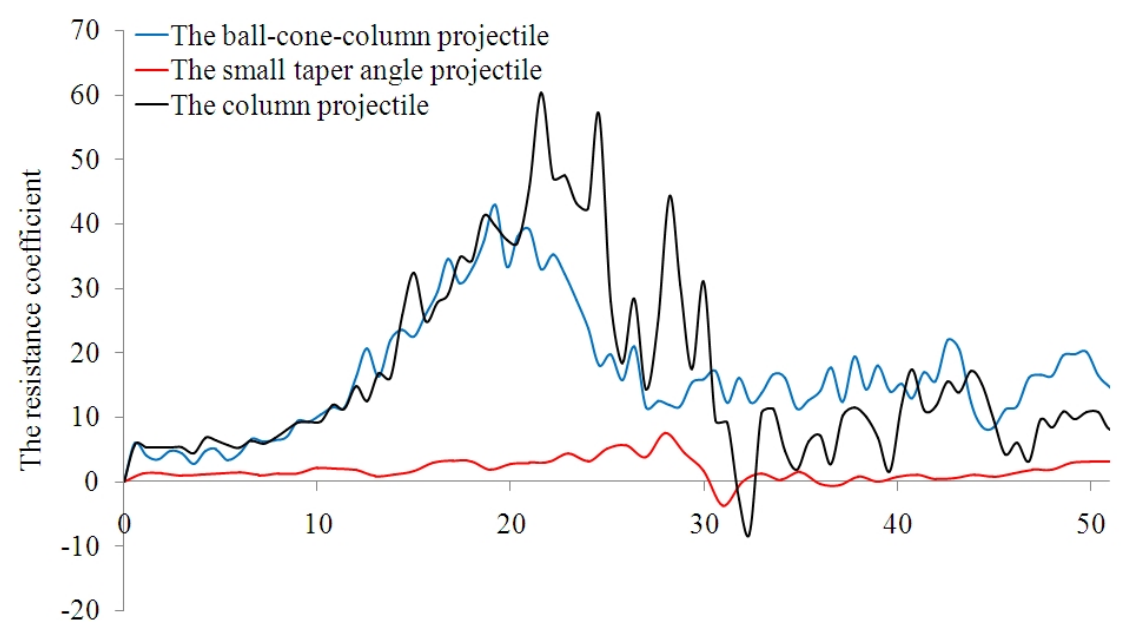

The time(ms)

Figure. 6 The variation of the resistance coefficient of the water-entry projectile with time

The results are shown in Fig. 6. The resistance coefficient of the three projectiles firstly increases and then decreases. This may be due that, during early stages, with the movement of the projectile, the contact between the projectile nose and water area gradually increased, so the resistance coefficient becomes larger. With the continuous movement of the projectile, the initial formation of the local cavity begins to expand, forming a relatively complete cavity around the projectile, reducing the contact area between the projectile and the water, so the resistance coefficient began to decrease. And then the resistance coefficient begins to oscillate more strongly because of the interaction between the projectile and the water, the contact area of the projectile and water changes constantly, so that the projectile ballistic fluctuations. As the speed of the projectile is constantly weakening, so the contact area should be increased, and the resistance coefficient should increase oscillatly. The resistance coefficient of the small taper angle projectile are smaller than the other kinds of projectiles.

The attack angle of the projectile is calculated, which is shown in Fig. 7. The small taper angle projectile has the smallest attack angle. All the three projectile have no attack angle adjustment process may becase they have no tail to adjust the attack angle. 


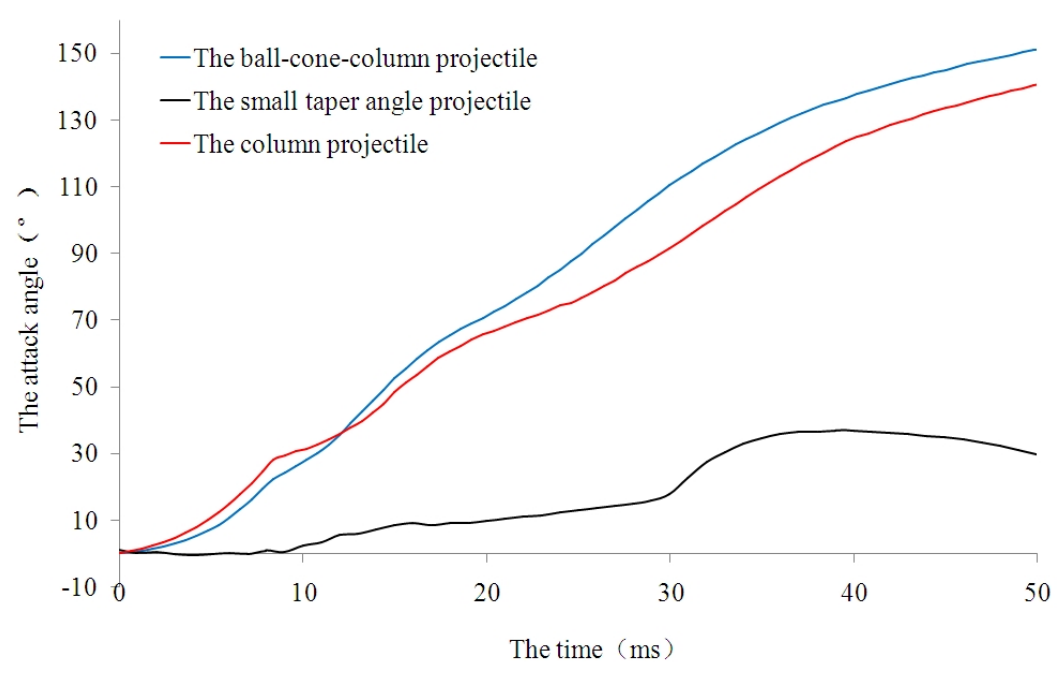

Fig. 7 The variation of the attack angle of the water-entry projectile with time

\section{conclusion}

By numerical simulation, the hydrodynamic characteristics, such as the displacement, velocity, acceleration, resistance coefficient and attack angle of the projectiles are analyzed. The small taper angle projectile has the smallest resistance coefficient and attack angle. The results of this study can provide some reference for the hydrodynamic shape design of the projectile penetrating water with high-speed.

\section{References}

[1] Gilbarg, D. and Anderson R A, Influence of atmospheric pressure on the phenomena accompanying the entry of spheres into water[J]. Journal of Applied Physics, 1948, 9(2):127-139.

[2] May A. and Woodhull J C, The virtual mass of a sphere entering water vertically[J]. Journal of Applied Physics, 1950, 21(12):1285-1289.

[3] ZHANG Wei,GUO Zitao,XIAO Xinke,et al, Experimental investigations on behaviors of projectile high-speed water entry[J]. Explosion and Shock Waves, 2011, 31(6):579-584. (in chinese)

[4] Ma Qing-peng;He Chun-tao;Wang Cong et al, Experimental investigation on vertical water-entry cavity of sphere[J]. Explosion and Shock Waves, 2014, 34(2): 174-180. (in chinese) 